\title{
POINCARÉ SERIES AND ZETA FUNCTION OF THE MONODROMY OF A QUASIHOMOGENEOUS SINGULARITY
}

\author{
W. Ebeling and S. M. Gusein-Zade
}

\begin{abstract}
We give a formula connecting the Poincaré series of the coordinate ring of a (Newton non-degenerate) quasihomogeneous hypersurface singularity with the Saito dual of the zeta function of its monodromy.
\end{abstract}

In the last years there have appeared several results which can be described as follows: the Poincaré series (or rather $a$ Poincaré series: sometimes one has to be careful with the choice) of the ring of functions on a singularity is connected with the zeta function of its classical monodromy transformation. The first observation of this sort [CDG1] says that the Poincare series of an irreducible plane curve singularity (with respect to the natural filtration defined by a uniformization of the curve) coincides with the zeta function of its classical monodromy transformation. There was obtained a global version of this statement for an algebraic plane curve with one place at infinity [CDG2]. For a reducible plane curve singularity, it was shown that the Poincaré polynomial (appropriately defined as a polynomial in several variables) of the multi-index filtration of the ring of functions (defined by uniformizations of the branches of the curve) coincides with the Alexander polynomial $\Delta\left(t_{1}, \ldots, t_{r}\right)$ (in several variables) of the link of the singularity [CDG3, CDG4]. (The Alexander polynomial reduces to the zeta function of the monodromy when identifying all the variables $t_{i}: \zeta(t)=\Delta(t, \ldots, t)$.) It was noticed that this statement has a formulation in terms similar to motivic integration [CDG5].

For an isolated quasihomogeneous two-dimensional hypersurface singularity, it was shown that the Poincaré series of the (quasihomogeneous) filtration on the ring of functions on the singularity can be obtained from the characteristic polynomial of the classical monodromy operator by applying the Saito duality construction and multiplying by binomials corresponding to the orbit invariants of the natural action of the group $\mathbb{C}^{*}$ of nonzero complex numbers on the surface [E1, E2]. Saito duality [S1, S2] was formulated in order to describe the connection between the characteristic polynomials of the dual singularities in Arnold's

Received March 18, 2002.

2000 Mathematics Subject Classification. 14B05, 32S40.

Keywords and phrases. quasihomogeneous hypersurface singularity, Poincaré series, zeta function of monodromy.

Partially supported by the DFG-programme "Global methods in complex geometry" (Eb 102/4-1), grants RFBR-01-01-00739, NWO-RFBR-047.008.005, INTAS-00-259. 
strange duality. So there it connected objects of the same nature. In [E1, E2] the Saito duality is used to connect objects of different nature: topological and algebraic ones.

The proofs of all the results essentially consist of computing both sides of the equations (in terms of a resolution or in terms of the weights of the variables) which turn out to coincide. Though each single one can be considered as a pure coincidence, the number of these "coincidences" shows that there must be a general phenomenon behind all of them which seems not to be understood. There is no proof which explains these coincidences.

The aim of the paper is to generalize the result of [E1] about quasihomogeneous surface singularities to any dimension and also to some non-isolated singularities. This generalization rewrites it in more uniform terms.

Let $f: \mathbb{C}^{n} \rightarrow \mathbb{C}$ be a quasihomogeneous polynomial function of degree $d$ with respect to the weights $q_{1}, \ldots, q_{n}\left(q_{i}\right.$ are positive integers, $\left.\operatorname{gcd}\left(q_{1}, \ldots, q_{n}\right)=1\right)$, i.e., $f\left(\lambda^{q_{1}} x_{1}, \ldots, \lambda^{q_{n}} x_{n}\right)=\lambda^{d} f\left(x_{1}, \ldots, x_{n}\right), \lambda \in \mathbb{C}$. This means that $f(x)=$ $\sum a_{k} x^{k}\left(x=\left(x_{1}, \ldots, x_{n}\right), k=\left(k_{1}, \ldots, k_{n}\right), x^{k}=x_{1}^{k_{1}} \cdot \ldots \cdot x_{n}^{k_{n}}\right)$ where, for $a_{k} \neq 0, q_{1} k_{1}+\ldots+q_{n} k_{n}=d$. The group $\mathbb{C}^{*}$ of nonzero complex numbers acts on the space $\mathbb{C}^{n}$ by $\lambda *\left(x_{1}, \ldots, x_{n}\right)=\left(\lambda^{q_{1}} x_{1}, \ldots, \lambda^{q_{n}} x_{n}\right)$.

Let $(X, 0)=\left(f^{-1}(0), 0\right)$ be the zero set of $f$, and let $A=\mathbb{C}[x] /(f)$ be the coordinate ring of $X$. There is a natural grading on the ring $A: A_{s}$ is the set of functions $g \in A$ such that $g(\lambda * x)=\lambda^{s} g(x)$. Let $P_{X}(t)=\sum_{s=0}^{\infty} \operatorname{dim} A_{s} t^{s}$ be the Poincaré series of the graded algebra $A=\oplus_{s=0}^{\infty} A_{s}$.

Let $X_{\varepsilon}=f^{-1}(\varepsilon) \cap B_{\delta}\left(0<\|\varepsilon\| \ll \delta, B_{\delta}\right.$ is the ball of radius $\delta$ centred at the origin in $\mathbb{C}^{n}$ ) be the Milnor fibre of the germ of the function $f$ at the origin, let $h: X_{\varepsilon} \rightarrow X_{\varepsilon}$ be the classical monodromy transformation of the germ $f$, and let $\widetilde{\zeta}_{f}(t)$ be its (reduced) zeta function:

$$
\widetilde{\zeta}_{f}(t)=\prod_{p \geq 0}\left\{\operatorname{det}\left(\mathrm{id}-\left.t \cdot h_{*}\right|_{\tilde{H}_{p}\left(X_{\varepsilon}\right)}\right)\right\}^{(-1)^{p}} .
$$

Pay attention that in the definition there are the reduced homology groups of the Milnor fibre $X_{\varepsilon}$, i.e., its homology groups modulo a point. If $X$ has an isolated singular point at the origin, $n \geq 2$, then

$$
\left(\widetilde{\zeta}_{f}(t)\right)^{(-1)^{n-1}}=\operatorname{det}\left(\mathrm{id}-\left.t \cdot h_{*}\right|_{H_{n-1}\left(X_{\varepsilon}\right)}\right)
$$

is the characteristic polynomial of the classical monodromy operator of $f$.

The zeta function $\widetilde{\zeta}_{f}(t)$ can be written in the form

$$
\prod_{\ell \mid d}\left(1-t^{\ell}\right)^{\alpha_{\ell}}, \alpha_{\ell} \in \mathbb{Z}
$$

Following K. Saito [S1, S2], we define the Saito dual (rational) function $\widetilde{\zeta}_{f}^{*}(t)$ by

$$
\widetilde{\zeta}_{f}^{*}(t)=\prod_{m \mid d}\left(1-t^{m}\right)^{-\alpha_{d / m}}
$$


Let $Y=(X \backslash\{0\}) / \mathbb{C}^{*}$ be the space of orbits of the $\mathbb{C}^{*}$-action on $X \backslash\{0\}$. There is a natural stratification of the variety $Y$ defined by the types of the orbits: $Y_{m}$ is the set of orbits for which the isotropy group is the cyclic group of order $m$. (The stratum $Y_{m}$ can be nonempty only if $m$ is equal to the greatest common divisor of a subset of the weights $q_{1}, \ldots, q_{n}$.)

Let $\Gamma$ be the Newton diagram of the germ $f$, i.e., the convex hull in $\mathbb{R}^{n}$ of the points $k \in \mathbb{Z}^{n}$ such that $a_{k} \neq 0\left(f(x)=\sum a_{k} x^{k}\right)$. The diagram $\Gamma$ lies in the hyperplane $H=\left\{k \in \mathbb{R}^{n} \mid q_{1} k_{1}+\ldots+q_{n} k_{n}=d\right\}$.

Suppose that the function $f$ is non-degenerate with respect to its Newton diagram $\Gamma$ in the sense of $[\mathrm{V}]$. For a topological space $Z$ let $\chi(Z)$ denote its Euler characteristic.

\section{Theorem 1.}

$$
P_{X}(t)=\widetilde{\zeta}_{f}^{*}(t) \cdot \prod_{m \geq 1}\left(1-t^{m}\right)^{-\chi\left(Y_{m}\right)}
$$

Remarks. 1. We put the correction term $\prod_{m \geq 1}\left(1-t^{m}\right)^{-\chi\left(Y_{m}\right)}$ on the same side as $\widetilde{\zeta}_{f}^{*}(t)$ because in some sense its nature is more close to the one of the zeta function.

2. For $n=2$, the statement reduces to the statement of [CDG1] for irreducible quasihomogeneous plane curve singularities (i.e., plane curve singularities with one Puiseux pair). In this case, the zeta function $\widetilde{\zeta}_{f}(t)$ is self dual and $Y=Y_{1}=$ pt. For isolated hypersurface singularities with $n=3$, the statement coincides with that of [E1]. The number of equal orbit invariants which participate in the equation of [E1] is the Euler characteristic of the corresponding 0-dimensional set, the exponent at the term $(1-t)$ is the Euler characteristic $\chi\left(Y_{1}\right)$ of the open stratum $Y_{1}$ of the quotient curve $Y$ where the $\mathbb{C}^{*}$-action is free.

Proof. One has

$$
P_{X}(t)=\frac{1-t^{d}}{\prod_{i=1}^{n}\left(1-t^{q_{i}}\right)} .
$$

For $I \subset I_{0}=\{1, \ldots, n\}$, let $|I|$ be the number of elements of $I, L_{I}=\{k \in$ $\mathbb{R}^{n} \mid k_{i}=0$ for $\left.i \notin I\right\}$, and $\Gamma_{I}=\Gamma \cap L_{I}$. Let $T_{I}=\left\{x \in \mathbb{C}^{n} \mid x_{i}=0\right.$ for $i \notin I, x_{i} \neq$ 0 for $i \in I\}$ be the complex torus of dimension $|I|$ and let $m_{I}=\operatorname{gcd}\left(q_{i}, i \in\right.$ $I)$. The integer $m_{I}$ is the order of the isotropy group of the $\mathbb{C}^{*}$-action on the intersection of $X$ with the torus $T_{I}$. From [V] it follows that

$$
\widetilde{\zeta}_{f}(t)=(1-t)^{-1} \prod_{I \subset I_{0},|I| \geq 1}\left(1-t^{d / \operatorname{gcd}\left(d, m_{I}\right)}\right)^{(-1)^{|I|-1}(|I|-1) ! V\left(\Gamma_{I}\right)}
$$

where $V\left(\Gamma_{I}\right)$ is the $(|I|-1)$-dimensional volume of the polyhedron $\Gamma_{I}$ defined by the lattice $H \cap L_{I} \cap \mathbb{Z}^{n}$ (i.e., the volume of a fundamental parallelepiped of it is equal to 1 by definition). For $|I|=1, V(\mathrm{pt})=1, V(\emptyset)=0$. Note that, if $m_{I} \neq \operatorname{gcd}\left(d, m_{I}\right)$, i.e., if $m_{I}$ does not divide $d$, then $\Gamma_{I}$ is empty. 
The Saito dual of $\widetilde{\zeta}_{f}(t)$ is equal to

$$
\widetilde{\zeta}_{f}^{*}(t)=\left(1-t^{d}\right) \prod_{I \subset I_{0},|I| \geq 1}\left(1-t^{m_{I}}\right)^{(-1)^{|I|}(|I|-1) ! V\left(\Gamma_{I}\right)} .
$$

For $|I| \geq 2,(-1)^{|I|}(|I|-1) ! V\left(\Gamma_{i}\right)$ is equal to the Euler characteristic of $(X \cap$ $\left.T_{I}\right) / \mathbb{C}^{*}$. For $\Gamma_{I} \neq \emptyset$ this follows from $[\mathrm{BKKh}, \mathrm{Kh}]$. For $\Gamma_{I}=\emptyset$, the intersection $V \cap T_{I}$ coincides with $T_{I}$ and $\chi\left(T_{I} / \mathbb{C}^{*}\right)=0$ (for $|I| \geq 2$ ). If $|I|=1$, i.e., for $I=\{i\}$ consisting of one element $i, 1 \leq i \leq n$, the intersection $X \cap T_{\{i\}}$ is empty if $\Gamma_{\{i\}}$ is not empty (i.e., is a point) and vice versa. The Euler characteristic of $T_{\{i\}} / \mathbb{C}^{*}=$ pt is equal to 1 . Therefore $\chi\left(\left(X \cap T_{\{i\}}\right) / \mathbb{C}^{*}\right)=1-V\left(\Gamma_{\{i\}}\right)$. Thus

$$
\widetilde{\zeta}_{f}^{*}(t)=\frac{1-t^{d}}{\prod_{i=1}^{n}\left(1-t^{q_{i}}\right)} \prod_{I \subset I_{0},|I| \geq 1}\left(1-t^{m_{I}}\right)^{\chi\left(\left(X \cap T_{I}\right) / \mathbb{C}^{*}\right)} .
$$

Since $Y_{m}=\bigcup_{I: m_{I}=m}\left(X \cap T_{I}\right) / \mathbb{C}^{*}$ and the Euler characteristic is additive,

$$
\widetilde{\zeta}_{f}^{*}(t)=P_{X}(t) \cdot \prod_{m \geq 1}\left(1-t^{m}\right)^{\chi\left(Y_{m}\right)}
$$

\section{References}

[BKKh] D. N. Bernstein, A. G. Kushnirenko, A. G. Khovanskii, Newton Polyhedra, Uspekhi Mat. Nauk 31 (1976), 201-202.

[CDG1] A. Campillo, F. Delgado, S. M. Gusein-Zade, On the monodromy of a plane curve singularity and the Poincaré series of its ring of functions, Funktsional. Anal. i Prilozhen. 33 (1999), 66-68; English translation in Funct. Anal. Appl. 33 (1999), $56-57$.

[CDG2] _ On the monodromy at infinity of a plane curve and the Poincaré series of its coordinate ring, Pontryagin Conference, 8, Topology (Moscow, 1998). J. Math. Sci. (New York) 105 (2001), 1839-1842.

[CDG3] _ The Alexander polynomial of a plane curve singularity and the ring of functions on the curve, Uspekhi Mat. Nauk 54 (1999), 157-158; English translation in Russian Math. Surveys 54 (1999), 634-635.

[CDG4] _ The Alexander polynomial of a plane curve singularity via the ring of functions on it, preprint (to appear in Duke Math. J.) : math.AG/0205111

[CDG5] _ Integration with respect to the Euler characteristic over a function space, and the Alexander polynomial of a plane curve singularity, Uspekhi Mat. Nauk 55 (2000), 127-128; English translation in Russian Math. Surveys 55 (2000), 1148-1149.

[E1] W. Ebeling, Poincaré series and monodromy of a two-dimensional quasihomogeneous hypersurface singularity, Manuscripta math. 107 (2002), 271-282.

[E2] The Poincaré series of some special quasihomogeneous surface singularities, preprint: math.AG/0004086

[Kh] A. G. Khovanskii, Newton polyhedra and the genus of complete intersections, Funktsional. Anal. i Prilozhen. 12 (1978), 51-61; English translation in Funct. Anal. Appl. 12 (1978), 38-46.

[S1] K. Saito, Duality for regular systems of weights: a précis, Topological field theory, primitive forms and related topics (Kyoto, 1996), 379-426, Progr. Math., 160, Birkhäuser Boston, Boston, MA, 1998.

[S2] _ Duality for regular systems of weights, Asian J. Math. 2 (1998), 983-1047. 
[V] A. N. Varchenko, Zeta-function of monodromy and Newton's diagram, Invent. Math. 37 (1976), 253-262.

Universität Hannover, Institut für Mathematik, Postfach 6009, D-30060 HanNOVER, GeRmany.

E-mail address: ebeling@math.uni-hannover.de

Moscow State University, Faculty of Mechanics and Mathematics, Moscow, 119992, Russia.

E-mail address: sabir@mccme.ru 